\title{
Anxiety and Depression among COVID Positive Frontline Health Care Workers in Nepal
}

\author{
Sushila Baral ${ }^{1}$, Pawan Pandeya ${ }^{1}$, Kalyan Sapkota ${ }^{2}$, Srijana Paudel ${ }^{3}$, \\ Jewan Pariyar ${ }^{2}$, Yadu Nath Baral ${ }^{3}$, Arzoo Neupane ${ }^{4}$, Rajesh Kumar Yadav ${ }^{5}$ \\ ${ }^{1}$ Manmohan Memorial Institute of Health Sciences, Kathmandu, Nepal \\ ${ }^{2}$ Bharatpur Hospital, Chitwan, Nepal, Chitwan, Nepal \\ ${ }^{3}$ National Academy of Medical Sciences, Kathmandu, Nepal \\ ${ }^{4}$ Sahid Gangalal Memorial Heart Center, Kathmandu, Nepal \\ ${ }^{5}$ BNMT Nepal, Kathmandu, Nepal \\ Corresponding Author: Sushila Baral
}

\begin{abstract}
Background: COVID-19, an emerging contagious disease had affected all over the world and become a global health threat. Lockdown and restriction had made the life of the health care workers troublesome and psychosocial problems are exaggerated. Moreover, health care workers are the frontline workers to deal with the COVID-19 patients; they are at high risk. Due to social stigma and hectic schedule of work had made their life more stressful. The study aimed to assess anxiety and depression and its associated factors among COVID-19 positive frontline health care workers during COVID-19 pandemic.
\end{abstract}

Methods: A web-based cross-sectional study was conducted among 323 COVID-19 positive health care workers working in different provinces of Nepal. The Google form was made by adopting GAD7 for anxiety and PHQ-9 for depression and made available to study population through various social media.

Results: The prevalence of anxiety and depression among the COVID-19 positive frontline health care worker was $39 \%$ and $35.5 \%$ respectively. The study showed significant association between

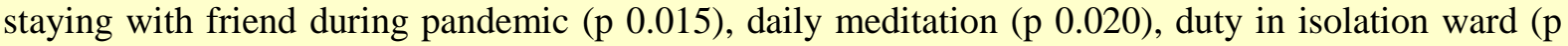
$0.027)$, unaware of incentives provision ( $\mathrm{p} 0.034)$, stigma $(\mathrm{p}<0.001)$ with anxiety and education status ( $\mathrm{p}$ 0.023), fear of dying from COVID-19 (p 0.045) and stigma ( $\mathrm{p}$ 0.05) with depression in multiple logistic regression analysis.

Conclusions: In the pandemic, COVID-19 positive frontline health workers had developed anxiety and depression symptoms. Training and sufficient preventive practice need to be implemented to control consequences of COVID 19 pandemic.

Keywords: Anxiety; Coronavirus; Depression; Frontline health workers, Nepal

\section{INTRODUCTION}

COVID-19 is a disease caused by the SARS-CoV-2 and first detected in Wuhan China in late December in 2019 which has spread all over the world. ${ }^{(1)}$ On 30 January 2020, the World Health Organization had declared the outbreak a public health emergency of international concern. ${ }^{(2)}$ COVID-19 disease has become a global health threat. ${ }^{(3)}$ According to WHO, the case fatality rate is around $2 \%{ }^{(4)}$ However, some reports suggest that the rate ranges from $0.3 \%$ to $0.6 \%{ }^{(5)}$ The COVID19 pandemic brought not only the risk of death from infection, but also unbearable psychological pressure. ${ }^{(6)}$ Study of China showed $16.5 \%$ depression while $28.8 \%$ anxiety symptoms. ${ }^{())}$Frontline health care workers involved in COVID-19 management were associated with a higher 
risk of symptoms of depression. ${ }^{(8)}$ A study conducted in India among health care workers showed $11.4 \%$ depression and $17.7 \%$ anxiety. ${ }^{(9)}$ Females, health professionals, staying alone were at high risk of anxiety and depression. ${ }^{(10)}$ Study of Nepal reflected $38 \%$ of the healthcare workers suffered from anxiety and/ or depression. ${ }^{(11)}$ More than half of health workers $(53.7 \%)$ reported that they faced stigma due to COVID-19. ${ }^{(12)}$

Study of TPO Nepal showed $37 \%$ suffered from anxiety and worry. ${ }^{(13)}$ Study among Nepalese nurses during COVID pandemic revealed that $25.6 \%, 10.3 \%$ and $16.7 \%$ suffered from moderate anxiety, severe anxiety and mild anxiety respectively. ${ }^{(14)}$ COVID-19 cases are increasing daily and many health care workers were infected and some of them died. Moreover, stigma towards the disease has also accelerated anxiety and increased stress. Thus, this study aims to assess anxiety, depression and associated factors that will guide interventions to maintain psychological well-being among the health care workers.

\section{MATERIAL AND METHODS}

Web based cross-sectional study was conducted to assess anxiety and depression among SARS CoV-2 positive frontline health care workers working in health institution of all provinces of Nepal. Nonprobability sampling method i.e. purposive sampling was done to reach to the study population. The duration of the study was from October to January with one month for data collection starting from first week of November.

\section{Participants}

Frontline health care workers working in the health institution and had been diagnosed as SARS-COV-2 positive were the most important selection criteria for study population. As the study is web based, the Google form was made available to study population through different social media.

\section{Sample size}

The Cochrane formula was used to calculated the sample size with anxiety prevalence $(p)=0.256$ among Nepalese nurses working in frontline during COVID$19^{(14)}$. Adding a $10 \%$ non-response rate, the final sample size for the study was 323 .

\section{Data collection tool}

Generalized Anxiety Disorder-7 tool was used to assess level of anxiety and Patient Health Questionnaire (PHQ-9) developed by PRIME MD was used to assess depression level among participants. ${ }^{(15)}$ GAD consists of 7 items on a four-point Likert scale ranging from 0 to 3 , in which 0 implies 'not at all' and 3 implies "nearly every day". The level of anxiety was categorized into four groups as minimal, mild, moderate and severe based on scoring $0-4,5-9,10-14$, and 15-21 respectively. Accordingly, minimal and mild were merged; ' $<10$ ' was absence of anxiety and moderate and severe were merged for presence of anxiety ' $\geq 10$ '. PHQ-9 consists of 9 items on a four-point Likert scale ranging from 0 to 3 , where 0 implies 'not at all' and 3 implies "nearly every day". The level of depression was categorized into five groups as minimal, mild, moderate, moderately severe and severe based on scoring 0-4, 5-9, 10-14, 15-19 and 20-27 respectively. Minimal and mild were merged ' $<10$ '; and moderate, moderately severe and severe were merged $' \geq 10$ '. The scores $(\geq 10)$ was used to determine the existence of depression. Internal validity was ensured through constructing the tools as per research objectives. Pre-tested tool was used for data collection.

\section{Data Analysis}

The collected data was saved and data cleaning was done. After data cleaning, it was imported to SPSS version 16 for the further processing and analysis of data. The descriptive analysis was done by expressing frequency and percentage. Also, based on the nature of data, both mean and median 
was computed. For inferential analysis, univariate and multiple logistic regression analysis was performed to find out the association between dependent and independent variables. Anxiety and depression levels were assessed by scoring. After scoring of general anxiety disorder and depression, its association with sociodemographic characteristics, health-related factors, work related factors and stigma was computed. Health care workers who had access to internet were only able to fill up the online Google form.

\section{Ethical consideration}

Ethical approval was taken from the Nepal Health Research Council (NHRC). Participants were fully informed regarding study objectives and written informed consent was obtained from participants previously to administration of questions in Google form. Confidentiality of the data was fully maintained and collected data was used only for the research purpose. All data was store in the computer database that was accessible only to the researcher with password protection and only share with the research team members. Monitoring was done regularly of the submitted forms and ensure accessibility to the participants of the research.

\section{RESULTS}

More than half $(51.7 \%)$ of the respondents were male. Most of the respondents belong to Hindu (86.4\%) religion followed by Buddhism (9.9\%). About $56.3 \%$ respondents had changes in their diet pattern. Three-fourth (77.4\%) respondents don't exercise or play sports daily. Two-third $(67.8 \%)$ respondents works in government health facility. More than half $(57 \%)$ respondents didn't have their own transportation to reach health facility. About $76.8 \%$ respondents don't feel that the workplace is well-equipped for COVID treatment. More than half (53.3\%) respondents didn't received training on COVID-19 prevention. Majority (91.6\%) didn't received psychosocial training.
Table 1: Sociodemographic Characteristics of the respondents

\begin{tabular}{|l|l|l|}
\hline Characteristics & Number & Frequency \\
\hline Provinces & & \\
\hline Province 1 & 40 & 12.4 \\
\hline Province 2 & 30 & 9.3 \\
\hline Bagmati & 92 & 28.5 \\
\hline Gandaki & 34 & 10.5 \\
\hline Lumbini & 53 & 16.4 \\
\hline Karnali & 36 & 11.1 \\
\hline Sudurpaschim & 38 & 11.8 \\
\hline Ethnicity & & \\
\hline Dalit & 32 & 9.9 \\
\hline Janajati & 71 & 22 \\
\hline Madhesi & 46 & 14.2 \\
\hline Brahmin/Chhetri & 174 & 53.9 \\
\hline Education & & \\
\hline Intermediate & 102 & 31.6 \\
\hline Bachelor & 139 & 43 \\
\hline Master and above & 82 & 25.4 \\
\hline Occupation & & \\
\hline Nursing & 126 & 39 \\
\hline Doctor & 98 & 30.3 \\
\hline Laboratory and diagnostics & 26 & 8 \\
\hline Pharmacy & 19 & 5.9 \\
\hline Health assistant & 42 & 13 \\
\hline Others & 12 & 3.7 \\
\hline Staying with during pandemic & & \\
\hline With family & 206 & 63.8 \\
\hline Without family & 87 & 26.9 \\
\hline With friends & 30 & 9.3 \\
\hline & & \\
\hline
\end{tabular}

Table 2 : Health-related factors of respondents

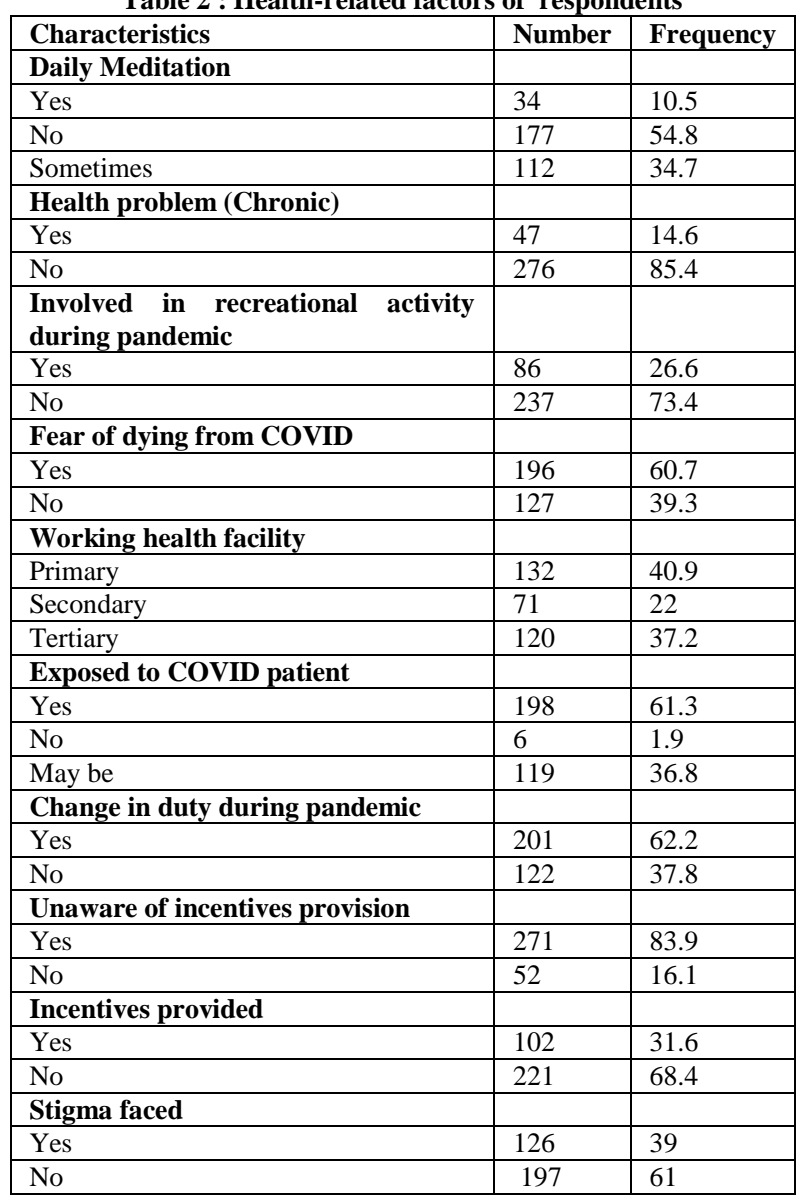

One-third of respondents had intermediate level education. More than one 
third $(39 \%)$ respondents were engaged in nursing profession. About two-third (64.7\%) were married and had a nuclear family. About $63.8 \%$ respondents were staying with family during the pandemic. More than half
$(55.7 \%)$ respondents live in their own home. Two-third (67.5\%) respondents had vulnerable population in their home. (Table 1)

Table 3: Univariate Logistic Regression Analysis with Anxiety

\begin{tabular}{|c|c|c|c|c|c|}
\hline \multirow{2}{*}{ Variables } & \multicolumn{2}{|l|}{ Anxiety } & \multirow{2}{*}{ p-value } & \multirow{2}{*}{ OR } & \multirow{2}{*}{$95 \%$ CI } \\
\hline & Presence & Absence & & & \\
\hline \multicolumn{6}{|l|}{ Provinces } \\
\hline Province 1 & $14(35 \%)$ & $26(65 \%)$ & $<<0.001^{*}$ & 1 & Ref \\
\hline Province 2 & $20(66.7 \%)$ & $10(33.3 \%)$ & & 3.71 & $1.36-10.08$ \\
\hline Bagmati & $10(10.9 \%)$ & $82(89.1 \%)$ & & 0.22 & $0.90-0.57$ \\
\hline Gandaki & $17(50 \%)$ & $17(50 \%)$ & & 1.85 & $0.72-4.73$ \\
\hline Lumbini & $28(52.8 \%)$ & $25(47.2 \%)$ & & 1.85 & $0.74-4.61$ \\
\hline Karnali & $18(50 \%)$ & $18(50 \%)$ & & 1.85 & $0.73-4.66$ \\
\hline Sudurpaschim & $19(50 \%)$ & $19(50 \%)$ & & 2.08 & $0.89-4.83$ \\
\hline \multicolumn{6}{|l|}{ Gender } \\
\hline Male & $61(36.5 \%)$ & $106(63.5 \%)$ & 0.344 & 1 & Ref \\
\hline Female & $65(41.7 \%)$ & $91(58.3 \%)$ & & 1.24 & $0.79-1.94$ \\
\hline \multicolumn{6}{|l|}{ Religion } \\
\hline Hinduism & $108(38.7 \%)$ & $171(61.3 \%)$ & 0.642 & 1 & Ref \\
\hline Buddhism & $13(40.6 \%)$ & $19(59.4 \%)$ & & 1.08 & $0.51-2.28$ \\
\hline Islam & $4(57.1 \%)$ & $3(42.9 \%)$ & & 2.11 & $0.46-9.61$ \\
\hline Christian & $1(20 \%)$ & $4(80 \%)$ & & 0.39 & $0.04-3.58$ \\
\hline \multicolumn{6}{|l|}{ Ethnicity } \\
\hline Dalit & $16(50 \%)$ & $16(50 \%)$ & $0.009^{*}$ & 1 & Ref \\
\hline Janajati & $23(32.4 \%)$ & $48(67.6 \%)$ & & 0.48 & $0.20-1.12$ \\
\hline Madhesi & $27(58.7 \%)$ & $19(41.3 \%)$ & & 1.42 & $0.57-3.52$ \\
\hline Brahmin/ Chhetri & $60(34.5 \%)$ & $114(65.5 \%)$ & & 0.52 & $0.24-1.12$ \\
\hline \multicolumn{6}{|l|}{ Place of residence } \\
\hline Rural municipality & $37(52.1 \%)$ & $34(47.9 \%)$ & $0.014^{*}$ & 1 & Ref \\
\hline Municipality & $55(41.4 \%)$ & $78(58.6 \%)$ & & 0.64 & $0.36-1.15$ \\
\hline Sub metropolitan & $12(30 \%)$ & $28(70 \%)$ & & 0.39 & $0.17-0.89$ \\
\hline Metropolitan & $22(27.8 \%)$ & $57(72.2 \%)$ & & 0.35 & $0.18-0.69$ \\
\hline \multicolumn{6}{|l|}{ Education } \\
\hline Intermediate & $41(40.2 \%)$ & $61(59.8 \%)$ & $0.001 *$ & 1 & Ref \\
\hline Bachelor & $67(48.2 \%)$ & $72(51.8 \%)$ & & 1.38 & $0.82-2.32$ \\
\hline Master and above & $18(22 \%)$ & $64(78 \%)$ & & 0.41 & $0.21-0.80$ \\
\hline \multicolumn{6}{|l|}{ Occupation } \\
\hline Nursing & $54(42.9 \%)$ & $72(57.1 \%)$ & 0.175 & 1 & Ref \\
\hline Doctor & $32(32.7 \%)$ & $66(67.3 \%)$ & & 0.64 & $0.37-1.12$ \\
\hline Laboratory and diagnostics & $11(42.3 \%)$ & $15(57.7 \%)$ & & 0.97 & $0.41-2.29$ \\
\hline Pharmacy & $10(52.6 \%)$ & $9(47.4 \%)$ & & 1.48 & $0.56-3.89$ \\
\hline Health Assistant & $18(42.9 \%)$ & $24(57.1 \%)$ & & 1.00 & $0.49-2.02$ \\
\hline Others & $1(8.3 \%)$ & $11(91.7 \%)$ & & 0.12 & $0.01-0.96$ \\
\hline \multicolumn{6}{|l|}{ Family Type } \\
\hline Nuclear & $91(43.5 \%)$ & $118(56.5 \%)$ & $0.024 *$ & 1 & Ref \\
\hline Joint & $35(30.7 \%)$ & $79(69.3 \%)$ & & 0.57 & $0.35-0.93$ \\
\hline \multicolumn{6}{|l|}{ Staying with during pandemic } \\
\hline With family & $77(37.4 \%)$ & $129(62.6 \%)$ & $0.020^{*}$ & 1 & Ref \\
\hline Without family & $30(34.5 \%)$ & $57(65.5 \%)$ & & 0.88 & $0.52-1.49$ \\
\hline With friends & $19(63.3 \%)$ & $11(36.7 \%)$ & & 2.89 & $1.31-6.40$ \\
\hline \multicolumn{6}{|l|}{ Exercise/ play sport } \\
\hline Yes & $12(16.4 \%)$ & $61(83.6 \%)$ & $<0.001 *$ & 1 & Ref \\
\hline No & $114(45.6 \%)$ & $136(54.4 \%)$ & & 4.26 & $2.19-8.30$ \\
\hline Daily Meditation & & & & & \\
\hline Yes & $2(5.9 \%)$ & $32(94.1 \%)$ & $0.003 *$ & 1 & Ref \\
\hline No & $74(41.8 \%)$ & $103(58.2 \%)$ & & 11.49 & $2.67-49.47$ \\
\hline Sometimes & $50(44.6 \%)$ & $62(55.4 \%)$ & & 12.90 & $2.94-56.47$ \\
\hline Involved in recreational ac & & & & & \\
\hline Yes & $19(22.1 \%)$ & $67(77.9 \%)$ & $\begin{array}{l}<0.001 * \\
\end{array}$ & 1 & Ref \\
\hline No & $107(45.1 \%)$ & $130(54.9 \%)$ & & 2.90 & $1.64-5.13$ \\
\hline Fear of dying from COVII & & & & & \\
\hline Yes & $94(48 \%)$ & $102(52 \%)$ & $<0.001 *$ & 1 & Ref \\
\hline No & $32(25.2 \%)$ & $95(74.8 \%)$ & & 0.37 & $0.24-0.59$ \\
\hline Working health facility & & & & & \\
\hline Primary & $62(47 \%)$ & $70(53 \%)$ & $<0.001^{*}$ & 1 & Ref \\
\hline Secondary & $34(47.9 \%)$ & $37(52.1 \%)$ & & 1.04 & $0.58-1.84$ \\
\hline Tertiary & $30(25 \%)$ & $90(75 \%)$ & & 0.37 & $0.22-0.64$ \\
\hline
\end{tabular}


Sushila Baral et.al. Anxiety and depression among COVID positive frontline health care workers in Nepal.

\begin{tabular}{|l|l|l|l|l|l|}
\hline \multicolumn{7}{|c}{ Table 3 continued... } & & & \\
\hline Own transport & & & & & \\
\hline Yes & $39(28.1 \%)$ & $100(71.9 \%)$ & $<0.001^{*}$ & 1 & Ref \\
\hline No & $87(47.3)$ & $97(52.7)$ & & 2.30 & $1.44-3.68$ \\
\hline Duty in isolation ward & & & & & \\
\hline Yes & $65(47.4 \%)$ & $72(52.6 \%)$ & $0.008^{*}$ & 1 & Ref \\
\hline No & $61(32.8 \%)$ & $125(67.2 \%)$ & & 0.54 & $0.34-0.85$ \\
\hline Change in duty during pandemic & & & & & \\
\hline Yes & $67(33.3 \%)$ & $134(66.7 \%)$ & $0.007^{*}$ & 1 & Ref \\
\hline No & $59(48.4 \%)$ & $63(51.6 \%)$ & & 1.87 & $1.18-2.97$ \\
\hline Unaware of incentives provision & & & & & \\
\hline Yes & $118(43.5 \%)$ & $153(56.5 \%)$ & $<0.001^{*}$ & 1 & Ref \\
\hline No & $8(15.4 \%)$ & $44(84.6 \%)$ & & 0.24 & $0.10-0.52$ \\
\hline No Access to PPE & & & & & \\
\hline Yes & $116(41.4 \%)$ & $164(58.6 \%)$ & $0.023^{*}$ & 1 & Ref \\
\hline No & $10(23.3 \%)$ & $33(76.7 \%)$ & & 0.43 & $0.20-0.90$ \\
\hline Well-equipped workplace for COVID-19 & & & & & \\
\hline Yes & $21(28 \%)$ & $54(72 \%)$ & $0.026^{*}$ & 1 & Ref \\
\hline No & $105(42.3 \%)$ & $143(57.7 \%)$ & & 1.89 & $1.08-3.32$ \\
\hline Training on COVID prevention & & & & & \\
\hline Yes & $73(48.3 \%)$ & $78(51.7 \%)$ & $0.001^{*}$ & 1 & Ref \\
\hline No & $53(30.8 \%)$ & $119(69.2 \%)$ & & 0.48 & $0.30-0.75$ \\
\hline Psychosocial training & & & & & \\
\hline Yes & $6(22.2 \%)$ & $21(77.8 \%)$ & 0.062 & 1 & Ref \\
\hline No & $120(40.5 \%)$ & $176(59.5 \%)$ & & 2.39 & $0.94-6.09$ \\
\hline Stigma & & & & & \\
\hline Yes & $68(54 \%)$ & $58(46 \%)$ & $<0.001^{*}$ & 2.81 & $1.76-4.47$ \\
\hline No & $58(29.4 \%)$ & $139(70.6 \%)$ & & 1 & Ref \\
\hline
\end{tabular}

Table 4 Uni-variate logistic regression analysis with Depression

\begin{tabular}{|c|c|c|c|c|c|}
\hline \multirow{2}{*}{ Variables } & \multicolumn{2}{|l|}{ Depression } & \multirow{2}{*}{ p-value } & \multirow{2}{*}{ OR } & \multirow{2}{*}{$95 \% \mathrm{CI}$} \\
\hline & Presence & Absence & & & \\
\hline \multicolumn{6}{|l|}{ Provinces } \\
\hline Province 1 & $10(25 \%)$ & $30(75 \%)$ & \multirow{7}{*}{$<0.001^{*}$} & 1 & Ref \\
\hline Province 2 & $15(50 \%)$ & $15(50 \%)$ & & 3.0 & $1.09-8.25$ \\
\hline Bagmati & $11(12 \%)$ & $81(88 \%)$ & & 0.40 & $0.15-1.05$ \\
\hline Gandaki & $15(44.1 \%)$ & $19(55.9 \%)$ & & 2.36 & $0.88-6.34$ \\
\hline Lumbini & $31(58.5 \%)$ & $22(41.5 \%)$ & & 2.18 & $0.83-5.71$ \\
\hline Karnali & $17(47.2 \%)$ & $19(52.8 \%)$ & & 2.68 & $1.01-7.07$ \\
\hline Sudurpaschim & $16(42.1 \%)$ & $22(57.9 \%)$ & & 4.22 & $1.71-10.40$ \\
\hline \multicolumn{6}{|l|}{ Gender } \\
\hline Male & $55(32.9 \%)$ & $112(67.1 \%)$ & \multirow{2}{*}{0.300} & 1 & Ref \\
\hline Female & $60(38.5 \%)$ & $96(61.5 \%)$ & & 1.27 & $0.81-2.01$ \\
\hline \multicolumn{6}{|l|}{ Ethnicity } \\
\hline Brahmin/ Chhetri & $58(33.3 \%)$ & $116(66.7 \%)$ & \multirow{4}{*}{$0.004 *$} & 1 & Ref \\
\hline Dalit & $20(62.5 \%)$ & $12(37.5 \%)$ & & 3.33 & $1.52-7.2$ \\
\hline Janajati & $18(25.4 \%)$ & $53(74.6 \%)$ & & 0.67 & $0.365-1.26$ \\
\hline Madhesi & $19(41.3 \%)$ & $27(58.7 \%)$ & & 1.40 & $0.72-2.74$ \\
\hline \multicolumn{6}{|l|}{ Marital Status } \\
\hline Married & $73(34.9 \%)$ & $136(65.1 \%)$ & 0.731 & 1 & Ref \\
\hline Unmarried & $42(36.8 \%)$ & $72(63.2 \%)$ & & 1.09 & $0.68-1.75$ \\
\hline \multicolumn{6}{|l|}{ Place of residence } \\
\hline Metropolitan & $19(24.1 \%)$ & $60(75.9 \%)$ & \multirow{4}{*}{$0.028 *$} & 1 & Ref \\
\hline Rural municipality & $34(47.9 \%)$ & $37(52.1 \%)$ & & 2.90 & $1.44-5.81$ \\
\hline Municipality & $47(35.3 \%)$ & $86(64.7 \%)$ & & 1.72 & $0.92-3.22$ \\
\hline Sub metropolitan & $15(37.5 \%)$ & $25(62.5 \%)$ & & 1.89 & $0.833-4.31$ \\
\hline \multicolumn{6}{|l|}{ Education } \\
\hline Master and above & $18(22 \%)$ & $64(78 \%)$ & $0.015^{*}$ & 1 & Ref \\
\hline Bachelor & $58(41.7 \%)$ & $81(58.3 \%)$ & & 2.54 & $1.36-4.74$ \\
\hline Intermediate & $39(38.2 \%)$ & $63(61.8 \%)$ & & 2.20 & $1.14-4.25$ \\
\hline \multicolumn{6}{|l|}{ Family Type } \\
\hline Joint & $30(26.3 \%)$ & $84(73.7 \%)$ & $0.010^{*}$ & 1 & \multirow[t]{2}{*}{$0.32-0.86$} \\
\hline Nuclear & $85(40.7 \%)$ & $124(59.3 \%)$ & & 0.52 & \\
\hline \multicolumn{6}{|c|}{ Staying with during pandemic } \\
\hline With family & $68(33 \%)$ & $138(67 \%)$ & $0.001^{*}$ & 1 & Ref \\
\hline Without family & $26(29.9 \%)$ & $61(70.1 \%)$ & & 0.86 & $0.50-1.48$ \\
\hline With friends & $21(70 \%)$ & $9(30 \%)$ & & 4.73 & $2.05-10.89$ \\
\hline \multicolumn{6}{|c|}{ Change in diet pattern } \\
\hline Yes & $76(41.8 \%)$ & $106(58.2 \%)$ & $0.009 *$ & 1 & \multirow[t]{3}{*}{$0.33-0.86$} \\
\hline No & $39(27.7 \%)$ & $102(72.3 \%)$ & & & \\
\hline & & & & 0.53 & \\
\hline
\end{tabular}




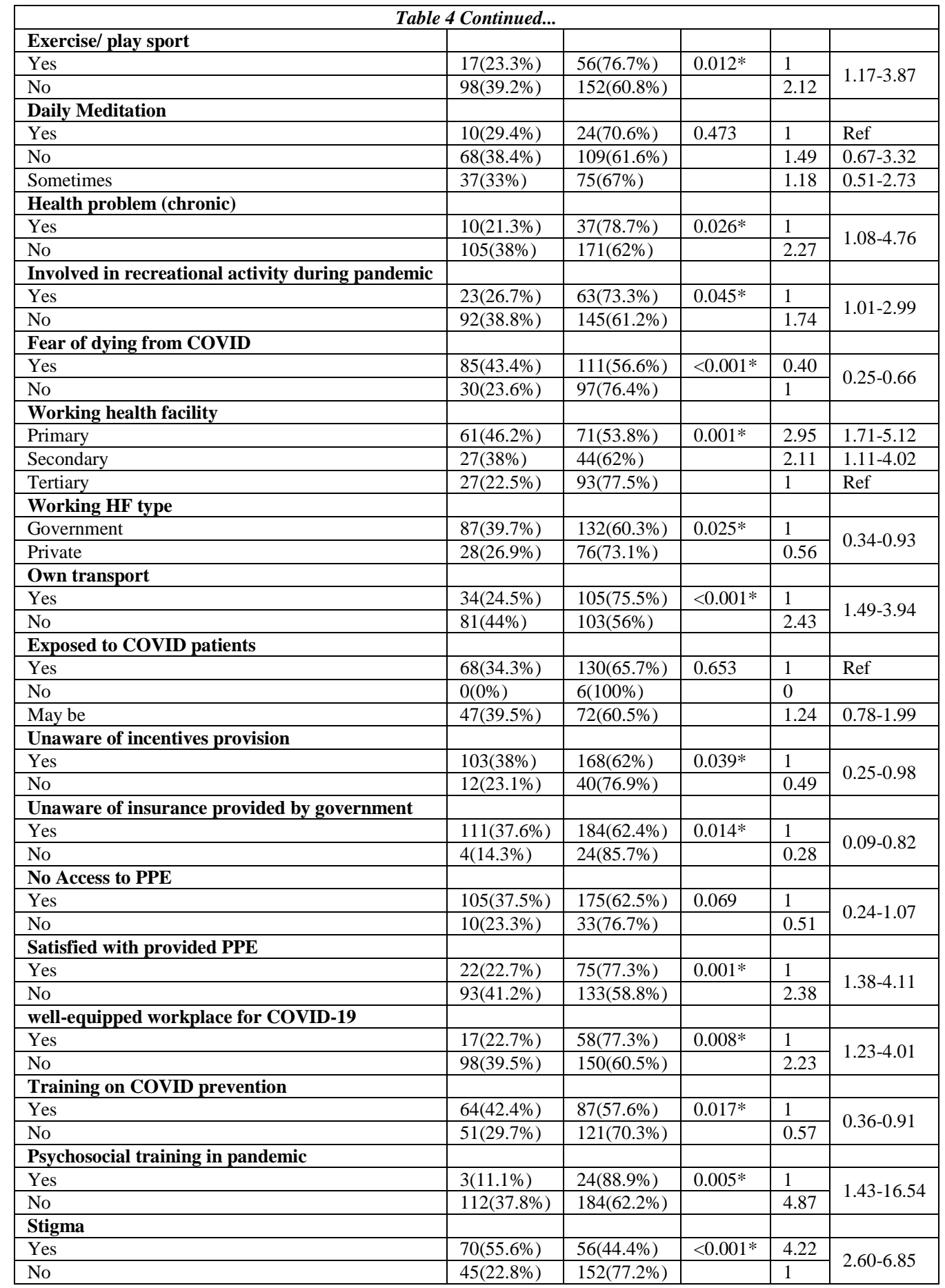

One in ten $(10.5 \%)$ respondents do meditation daily. About $85.4 \%$ respondents don't have chronic health problems. About three-fourth $(73.4 \%)$ respondents had involved in recreational activity during pandemic. About $60.7 \%$ respondents had fear of dying from COVID-19. Two-fifth $(40.9 \%)$ respondents were engaged in primary level health facility. About $61.3 \%$ respondents were exposed to COVID-19 patients. More than two-fifth $(42.4 \%)$ of the respondents had duty in isolation ward. Most of the respondents $(91.3 \%)$ were unaware of insurance provided by government. About $86.7 \%$ respondents responded for no access to PPE and around three-fourth $(70 \%)$ respondents weren't satisfied with provided PPE is illustrated in Table 2. 
In table 3, the univariate logistic regression analysis, the different factors like province $(\mathrm{p}<0.001)$, ethnicity ( $\mathrm{p} 0.009)$, place of residence ( $\mathrm{p} 0.014)$, education $(\mathrm{p}$ $0.001)$, family type (p 0.024), staying with (p 0.020), doing exercise/play sport ( $\mathrm{p}<$ 0.001 ), daily meditation ( $\mathrm{p} 0.003)$, involved in recreational activity during pandemic $(\mathrm{p}<$ 0.001), Fear of dying from COVID-19 ( $\mathrm{p}<$ $0.001)$, working health facility $(\mathrm{p}<0.001)$, own transportation facility to reach $\mathrm{HF}$ ( $\mathrm{p}<$ 0.001 ), duty in isolation ward ( $p$ 0.008), change in duty during pandemic ( $\mathrm{p} 0.007$ ), unaware of incentives provision $(\mathrm{p}<0.001)$, no access to PPE (p 0.023), well equipped workplace for COVID-19 (p 0.026), Training on COVID prevention ( $\mathrm{p}$ 0.001) were found statistically significantly associated with anxiety.

The factors like province $(\mathrm{p}<$ 0.001), ethnicity ( $\mathrm{p}$ 0.004), place of residence ( $p$ 0.028), education ( $p$ 0.015), family type ( $\mathrm{p} 0.010$ ), staying with during pandemic ( $\mathrm{p}$ 0.001), change in diet pattern (p 0.009), exercise/play sport (p 0.012),

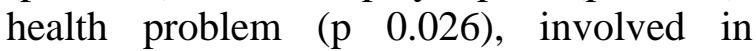
recreational activity ( $\mathrm{p} 0.045$ ), fear from dying from COVID-19 ( $\mathrm{p}<0.001$ ), working health facility ( $\mathrm{p}$ 0.001), type of working HF ( $\mathrm{p}$ 0.025), own transport ( $\mathrm{p}<0.001$ ), unaware of incentives provision ( $\mathrm{p}$ 0.039), unaware of insurance provided by government ( $\mathrm{p}$ 0.014), satisfied with provided PPE ( $\mathrm{p}$ 0.001), well equipped workplace for COVID-19 (p 0.008), training on COVID-19 prevention (p 0.017), psychosocial training in pandemic ( $\mathrm{p}$ 0.005) were statistically associated with depression. (Table 4) The predictors for anxiety (Table 5 ) and depression (Table 6) were shown.

Figure 1 explains one in fourth $(25.1 \%)$ respondents had minimal anxiety symptoms and around one in fourth (23.2\%) had minimal depressive symptoms. The prevalence of anxiety and depression was 39 $\%$ and $35.5 \%$ respectively.

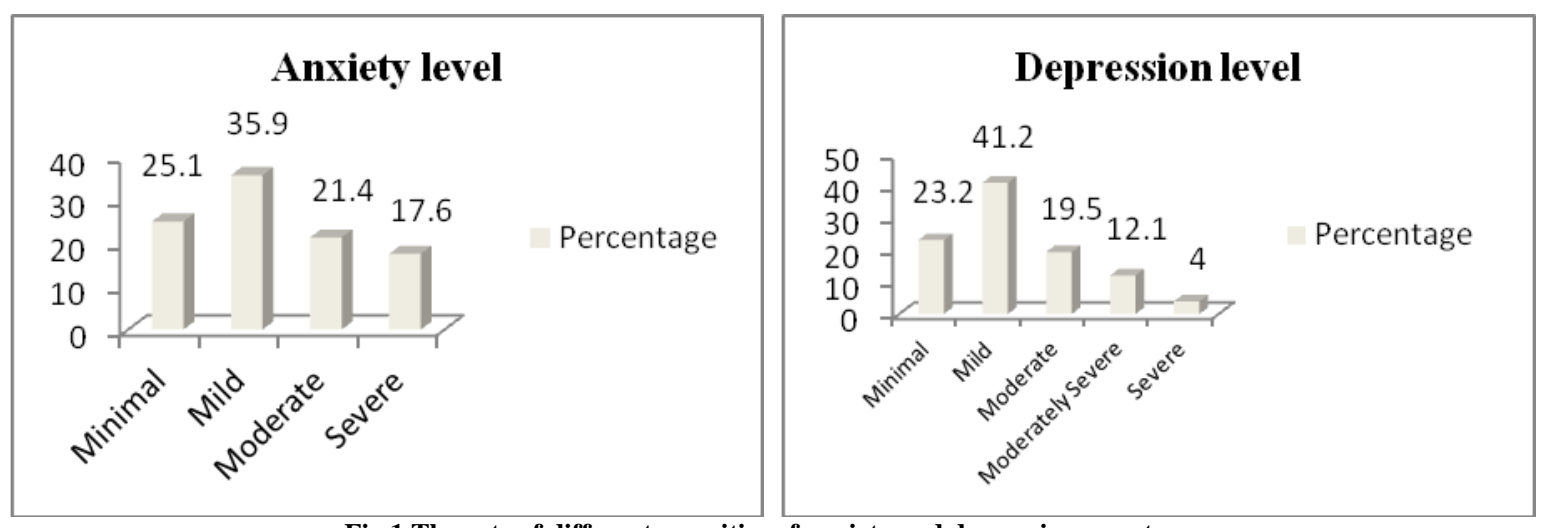

Fig 1 The rate of different severities of anxiety and depressive symptoms

Table 5 Multiple logistic regression analysis with anxiety

\begin{tabular}{|l|l|l|l|}
\hline Anxiety Variables & AOR & $\mathbf{9 5 \%}$ CI & P value \\
\hline $\begin{array}{l}\text { Staying with during } \\
\text { pandemic }\end{array}$ & & & \\
\hline With family & 1 & Ref & $0.015^{*}$ \\
\hline Without family & 0.92 & $0.45-1.90$ & \\
\hline With friends & 6.31 & $1.69-23.50$ & \\
\hline Daily Meditation & & & \\
\hline Yes & 1 & Ref & $0.020^{*}$ \\
\hline No & 21.29 & $2.46-184.22$ & \\
\hline Sometime & 19.94 & $2.32-171.05$ & \\
\hline Duty in isolation ward & & & \\
\hline Yes & 1 & Ref & $0.027^{*}$ \\
\hline No & 0.46 & $0.23-0.91$ & \\
\hline $\begin{array}{l}\text { Unaware of incentives } \\
\text { provision }\end{array}$ & & & \\
\hline Yes & 1 & Ref & $0.034^{*}$ \\
\hline No & 0.25 & $0.07-0.90$ & \\
\hline Stigma & & & \\
\hline No & 1 & Ref & $<0.001^{*}$ \\
\hline Yes & 3.16 & $1.66-6.03$ & \\
\hline & & & \\
\hline
\end{tabular}

Table 6 Multiple logistic regression analysis with depression

\begin{tabular}{|l|l|l|l|}
\hline Depression Variables & AOR & 95\% CI & P value \\
\hline Education status & & & \\
\hline Intermediate & 1 & Ref & $0.023^{*}$ \\
\hline Bachelor & 3.05 & $1.37-6.80$ & \\
\hline Master and above & 2.56 & $0.76-8.59$ & \\
\hline Fear of dying of COVID-19 & & & \\
\hline Yes & 1 & Ref & $0.045^{*}$ \\
\hline No & 0.48 & $0.23-0.98$ & \\
\hline Stigma & & & \\
\hline No & 1 & Ref & \\
\hline Yes & 2.54 & $1.32-4.88$ & $0.05^{*}$ \\
\hline
\end{tabular}

\section{DISCUSSION}

The study revealed that two-fifth $(39 \%)$ and more than one-third $(35.5 \%)$ COVID-19 positive frontline health care workers who had internet access were 
suffering from moderate to severe anxiety and depressive symptoms respectively. More than half of health workers $(53.7 \%)$ reported that they faced stigma due to COVID-19 which is higher than our study $(39 \%){ }^{(12)}$ This study shows $30 \%$ of the health workers were satisfied with provided PPE which is comparatively higher than the study conducted in Nepal among health workers $(21.1 \%)$ during COVID-19. ${ }^{(12)}$ More than half $(60.7 \%)$ of the frontline health works had fear of dying from COVID-19 in the present study while in another study conducted in Nepal showed $46.1 \%$ expressed high degree of COVID related fear. ${ }^{(12)}$ The study found different factors like daily meditation, duty in isolation ward, unaware of incentives provision were associated with anxiety whereas, strong association was found between education status and fear of dying from COVID-19 with depression.

\section{Anxiety}

Our study showed $25.1 \%$ had minimal anxiety, $35.9 \%$ had mild anxiety symptoms, $21.4 \%$ had observed among health care workers in minimal $(62.7 \%)$ and lower percent points in mild $(27.3 \%)$, moderate $(8 \%)$ and severe $(0.7 \%)$ anxiety symptoms in Nepal. ${ }^{(11)}$ Our study revealed prevalence of anxiety (39\%) was somehow lower than the study where anxiety $(41.9 \%)$ was found among health care workers during early phase of COVID-19 in Nepal. ${ }^{(12)}$ In the earlier phase, health workers were less informed about the pandemic. Study done in China anxiety among health workers was higher $(44.6 \%)$ than present study. Similar finding was found in a study done in Nepal which showed 38\% anxiety among health care workers on COVID-19 duty. ${ }^{(5)}$

\section{Depression}

The present study showed 23.2, $41.2,19.5,12.1,4.1$ percent were scored in minimal, mild, moderate moderately depressive and severely depressive symptoms respectively among the COVID-
19 positive health care workers. With contrast to our findings, $66 \%, 26 \%, 5.3 \%$, $0.7 \%$ and $2 \%$ COVID-19 positive health care workers had minimal, mild, moderate, moderate severe and severe depressive symptoms respectively. ${ }^{(11)}$ Study shows somehow lower depression $(35.6 \%)$ than the study conducted among health care workers during early phase of COVID-19 in Nepal $(37.5 \%) .{ }^{(12)}$ Another contradicts findings was found in the study done in China which showed about half $(50.4 \%)$ of health care workers suffered from depressive symptoms. ${ }^{(8)}$ While only $8 \%$ depressive symptom was reflected by the study conducted in Nepal. ${ }^{(11)}$

Our study shows that marital status (OR 0.65; 95\% CI, 0.40-1.05) and presence of chronic illness (OR 1.81; 95\% CI, 0.923.59) were not found to be statistically significant with anxiety while the study conducted in Ethiopia showed statistically significant with marital status (OR 3.56; $95 \% \mathrm{CI}, 2.30,6.38)$ and presence of chronic illness (OR 3.43; 95\% CI, 1.59-7.43). The present study reflects no PPE access (OR $0.43 ; 95 \%$ CI, 0.20-0.90) to be statistically significant with anxiety which is found similar with the study (OR, 2.55; 95\% CI, $1.43,4.56){ }^{(16)}$

\section{Limitations}

The present study had many limitations. Firstly, frontline health care workers who had access to internet were only able to fill up the online Google form. Secondly, self-reported responses were considered to assess anxiety and depression. Despite of all the limitations, study had made an important role to identify various factors as a predictor for anxiety and depression.

\section{CONCLUSIONS}

In the pandemic COVID 19 positive frontline health workers had developed anxiety and depression symptoms. Factors like staying with during pandemic, daily meditation, duty in isolation ward, unaware of incentives provision, stigma are found to 
be independent predictors for anxiety whereas educational status, fear of dying of COVID-19, stigma are the independent predictors for depression. Anxiety among health workers could be minimized by organizing meditation class. Motivational package needs to be developed for staffs working in isolation ward. Awareness program to minimize stigma in the community could prevent depression among health workers. Training and sufficient preventive practice need to be implemented to control consequences of COVID 19 pandemic.

\section{ACKNOWLEDGEMENT}

We are deeply grateful to all the participants who participated and provided their valuable time to fill up the Google form.

\section{Conflict of Interest: None}

\section{Source of Funding: None}

\section{Ethical Approval: Approved}

\section{REFERENCES}

1. Nishiura H, Jung S, Linton NM, Kinoshita R, Yang Y, Hayashi K, et al. The Extent of Transmission of Novel Coronavirus in Wuhan, China, 2020. J Clin Med [Internet]. 2020 Feb [cited 2020 Jul 3];9(2):330. Available from: https://www.mdpi.com/2077-0383/9/2/330

2. Mahase E. China coronavirus: WHO declares international emergency as death toll exceeds 200. BMJ. 2020 Jan 31;368:m408. Available from: https://doi.org/10.1136/bmj.m408

3. Anderson RM, Heesterbeek $\mathrm{H}$, Klinkenberg D, Hollingsworth TD. How will countrybased mitigation measures influence the course of the COVID-19 epidemic? Lancet Lond Engl [Internet]. 2020 [cited 2021 Jan 21];395(10228):931-4. Available from: https://www.ncbi.nlm.nih.gov/pmc/articles/ PMC7158572/

4. Timeline: WHO's COVID-19 response [Internet]. [cited 2021 Jan 21]. Available from: https://www.who.int/emergencies/diseases/n ovel-coronavirus-2019/interactive-timeline

5. Nishiura H, Kobayashi T, Yang Y, Hayashi K, Miyama T, Kinoshita R, et al. The Rate of Underascertainment of Novel Coronavirus (2019-nCoV) Infection: Estimation Using Japanese Passengers Data on Evacuation Flights. J Clin Med [Internet]. 2020 Feb 4 [cited 2020 Jul 3];9(2). Available from: https://www.ncbi.nlm.nih.gov/pmc/articles/ PMC7074297/

6. mental-health-considerations.pdf [Internet]. [cited 2021 Jan 21]. Available from: https://www.who.int/docs/defaultsource/coronaviruse/mental-healthconsiderations.pdf

7. Wang C, Pan R, Wan X, Tan Y, Xu L, Ho $\mathrm{CS}$, et al. Immediate Psychological Responses and Associated Factors during the Initial Stage of the 2019 Coronavirus Disease (COVID-19) Epidemic among the General Population in China. Int J Environ Res Public Health [Internet]. 2020 Mar 6 [cited 2021 Jan 21];17(5):1729. Available from: https://www.mdpi.com/16604601/17/5/1729

8. Lai J, Ma S, Wang Y, Cai Z, Hu J, Wei N, et al. Factors Associated With Mental Health Outcomes Among Health Care Workers Exposed to Coronavirus Disease 2019. JAMA Netw Open [Internet]. 2020 Mar 23 [cited 2021 Jan 21];3(3):e203976. Available from: https://jamanetwork.com/journals/jamanetw orkopen/fullarticle/2763229

9. Wilson W, Raj JP, Rao S, Ghiya M, Nedungalaparambil NM, Mundra $\mathrm{H}$, et al. Prevalence and Predictors of Stress, anxiety, and Depression among Healthcare Workers Managing COVID-19 Pandemic in India: A Nationwide Observational Study. Indian J Psychol Med [Internet]. 2020 Jul 1 [cited 2021 Jan 21];42(4):353-8. Available from: https://doi.org/10.1177/0253717620933992

10. Sigdel A, Bista A, Bhattarai N, Pun BC, Giri G, Marqusee $H$, et al. Depression, Anxiety and Depression-anxiety comorbidity amid COVID-19 Pandemic: An online survey conducted during lockdown in Nepal [Internet]. Public and Global Health; 2020 May [cited 2021 Jan 21]. Available from:

http://medrxiv.org/lookup/doi/10.1101/2020 .04 .30 .20086926 
11. Gupta AK, Mehra A, Niraula A, Kafle K, Deo SP, Singh B, et al. Prevalence of anxiety and depression among the healthcare workers in Nepal during the COVID-19 pandemic. Asian J Psychiatry [Internet]. 2020 Dec [cited 2021 Jan 21];54:102260. Available from: https://www.ncbi.nlm.nih.gov/pmc/articles/ PMC7313505/

12. Khanal P, Devkota N, Dahal M, Paudel K, Joshi D. Mental health impacts among health workers during COVID-19 in a low resource setting: a cross-sectional survey from Nepal. Glob Health [Internet]. 2020 Sep 25 [cited 2021 Jan 20];16(1):89. Available from: https://doi.org/10.1186/s12992-020-00621-z

13. Lockdown casts a long shadow on people's mental health: Nationwide survey - TPO Nepal [Internet]. [cited 2021 Jan 21]. Available from: http://tponepal.org/lockdown-casts-a-longshadow-on-peoples-mental-healthnationwide-survey/

14. Karki P, Katwal GBJ, Chandra A, Chandra A. Prevalence and Measurement of Anxiety and Depression in Nurses During Covid Pandemic in Nepal [Internet]. In Review;
2020 Jun [cited 2021 Jan 21]. Available from:

https://www.researchsquare.com/article/rs34462/v1

15. Spitzer RL, Kroenke K, Williams JBW, Löwe B. A Brief Measure for Assessing Generalized Anxiety Disorder: The GAD-7. Arch Intern Med [Internet]. 2006 May 22 [cited 2021 Jan 18];166(10):1092. Available from:

http://archinte.jamanetwork.com/article.asp $\mathrm{x}$ ?doi=10.1001/archinte.166.10.1092

16. Kibret S, Teshome D, Fenta E, Hunie M, Tamire T. Prevalence of anxiety towards COVID-19 and its associated factors among healthcare workers in a Hospital of Ethiopia. Tu W-J, editor. PLOS ONE [Internet]. 2020 Dec 8 [cited 2021 Jan 21];15(12):e0243022. Available from: https://dx.plos.org/10.1371/journal. pone. 0243022

How to cite this article: Baral S, Pandeya P, Sapkota K et.al. Anxiety and depression among COVID positive frontline health care workers in Nepal. Int J Health Sci Res. 2021; 11(4):83-92. DOI: https://doi.org/10.52403/ijhsr.20210411 